\title{
Single breath-hold saturation recovery 3D cardiac T1 mapping via compressed SENSE at $3 \mathrm{~T}$
}

\author{
Tiago Ferreira da Silva ${ }^{1,2} \cdot$ Carlos Galan-Arriola ${ }^{2,3} \cdot$ Paula Montesinos ${ }^{1} \cdot$ Gonzalo Javier López-Martín ${ }^{2}$. \\ Manuel Desco ${ }^{4,5,6,7} \cdot$ Valentín Fuster $^{2,8} \cdot$ Borja Ibáñez $^{2,3,9} \cdot$ Javier Sanchez-Gonzalez ${ }^{1}$ (1)
}

Received: 21 January 2020 / Revised: 21 April 2020 / Accepted: 25 April 2020 / Published online: 14 May 2020

(c) The Author(s) 2020

\begin{abstract}
Objectives To propose and validate a novel imaging sequence that uses a single breath-hold whole-heart 3D T1 saturation recovery compressed SENSE rapid acquisition (SACORA) at 3T.

Methods The proposed sequence combines flexible saturation time sampling, compressed SENSE, and sharing of saturation pulses between two readouts acquired at different RR intervals. The sequence was compared with a 3D saturation recovery single-shot acquisition (SASHA) implementation with phantom and in vivo experiments (pre and post contrast; 7 pigs) and was validated against the reference inversion recovery spin echo (IR-SE) sequence in phantom experiments.

Results Phantom experiments showed that the T1 maps acquired by 3D SACORA and 3D SASHA agree well with IR-SE. In vivo experiments showed that the pre-contrast and post-contrast T1 maps acquired by 3D SACORA are comparable to the corresponding 3D SASHA maps, despite the shorter acquisition time (15s vs. 188s, for a heart rate of $60 \mathrm{bpm})$. Mean septal pre-contrast T1 was $1453 \pm 44 \mathrm{~ms}$ with 3D SACORA and $1460 \pm 60 \mathrm{~ms}$ with 3D SASHA. Mean septal post-contrast T1 was $824 \pm 66 \mathrm{~ms}$ and $824 \pm 60 \mathrm{~ms}$.

Conclusion 3D SACORA acquires 3D T1 maps in 15 heart beats (heart rate, $60 \mathrm{bpm}$ ) at 3T. In addition to its short acquisition time, the sequence achieves good $\mathrm{T} 1$ estimation precision and accuracy.
\end{abstract}

Electronic supplementary material The online version of this article (https://doi.org/10.1007/s10334-020-00848-2) contains supplementary material, which is available to authorized users.

Borja Ibáñez

bibanez@cnic.es

$\checkmark$ Javier Sanchez-Gonzalez

Javier.Sanchez.Gonzalez@philips.com

Tiago Ferreira da Silva

Tiago.Ferreira@philips.com

Carlos Galan-Arriola

carlos.galan@cnic.es

Paula Montesinos

Paula.Montesinos@philips.com

Gonzalo Javier López-Martín

gonzalojavier.lopez@cnic.es

Manuel Desco

desco@hggm.es

Valentín Fuster

vfuster@cnic.es

1 Philips Healthcare Iberia, Madrid, Spain
2 Centro Nacional de Investigaciones Cardiovasculares Carlos III (CNIC), Madrid, Spain

3 CIBER de Enfermedades Cardiovasculares (CIBERCV), Madrid, Spain

4 Unidad de imagen avanzada, Centro Nacional de Investigaciones Cardiovasculares Carlos III (CNIC), Madrid, Spain

5 CIBER de salud mental (CIBERSAM), Madrid, Spain

6 Departamento de Bioingeniería e Ingeniería Aerospacial, Universidad Carlos III, Madrid, Spain

7 Medicina y Cirugía Experimental, Instituto de Investigacion Sanitaria Gregorio Marañón, Madrid, Spain

8 The Zena and Michael A. Wiener CVI, Icahn School of Medicine at Mount Sinai, New York, USA

9 IIS-Fundación Jiménez Díaz University Hospital, Madrid, Spain 
Keywords Cardiovascular magnetic resonance - Tissue characterization · 3D cardiac T1 mapping - Single breathhold $\cdot$ Saturation recovery

$\begin{array}{ll}\text { Abbreviations } \\ \text { CMR } & \text { Cardiovascular magnetic resonance } \\ \text { T1 } & \text { Longitudinal relaxation time } \\ \text { 2D } & \text { Two-dimensional } \\ \text { MOLLI } & \text { Modified Look-Locker inversion recovery } \\ \text { SSFP } & \text { Steady-state free precession } \\ \text { SASHA } & \text { Saturation recovery single-shot acquisition } \\ \text { SNR } & \text { Signal-noise ratio } \\ \text { MLLSR } & \text { Modified Look-Locker acquisition using } \\ & \text { saturation recovery } \\ \text { SAPPHIRE } & \text { Saturation pulse-prepared heart rate inde- } \\ & \text { pendent inversion recovery } \\ \text { 3D } & \text { Three-dimensional } \\ \text { LV } & \text { Left ventricle } \\ \text { FLASH } & \text { Fast low angle shot } \\ \text { T2 } & \text { Transverse relaxation time } \\ \text { SENSE } & \text { Sensitivity encoding } \\ \text { SACORA } & \text { Saturation recovery compressed SENSE } \\ & \text { rapid acquisition } \\ \text { HR } & \text { Heart rate } \\ \text { MRI } & \text { Magnetic resonance imaging } \\ \text { PD } & \text { Proton density } \\ \text { TS } & \text { Saturation time } \\ \text { TFE } & \text { Turbo field echo } \\ \text { TR } & \text { Repetition time } \\ \text { TE } & \text { Echo time } \\ \text { FA } & \text { Flip angle } \\ \text { RF } & \text { Radiofrequency } \\ \text { IR-SE } & \text { Inversion recovery spin-echo } \\ \text { ROI } & \text { Region of interest } \\ \text { CV } & \text { Coefficient of variation } \\ \text { MR } & \text { Magnetic resonance } \\ \text { AHA } & \text { American heart association } \\ \text { Cl } & \text { Confidence interval } \\ & \end{array}$

\section{Introduction}

Cardiovascular magnetic resonance (CMR) is a medical imaging modality whose wide applications include tissue characterization across different physiological and pathological conditions. CMR longitudinal relaxation time (T1) mapping has become particularly relevant since it differs between healthy and diseased tissue, and aims to play an important role in clinical decision-making in cardiovascular disease [1-3]. T1 mapping provides pixel-wise $\mathrm{T} 1$ values by fitting images acquired during the T1 magnetization recovery at different times after application of an inversion pulse [4], a saturation pulse [5], or a combination of both [6].
CMR T1 mapping is complex due to the heart's motion, and several two-dimensional (2D) pulse sequences have been presented in recent years to tackle the problem [7-9]. However, these sequences are designed to acquire only a single slice per breath-hold. The modified Look-Locker inversion recovery (MOLLI) sequence applies an inversion pulse followed by different single-shot steady-state free precession (SSFP) readouts over multiple heartbeats [4]. MOLLI is precise and reproducible; however, it underestimates T1, mainly due to the magnetization transfer effect and imperfect inversion efficiency [8]. To overcome this limitation, saturation recovery single-shot acquisition (SASHA) replaces the inversion pulses with saturation pulses, thus avoiding the underestimation of $\mathrm{T} 1$ values due to incomplete recovery of the signal after the inversion pulse [5]. This approach improves accuracy at the cost of a lower signal-noise ratio (SNR). A different 2D saturation recovery sequence design is provided by modified Look-Locker acquisition using saturation recovery (MLLSR), which allows the saturation pulses to be shared between several readouts acquired in different heart beats to minimize T1 estimation error and provide high flexibility [10]. Lastly, saturation pulse-prepared heart rate independent inversion recovery (SAPPHIRE) is a hybrid approach that uses inversion and saturation pulses and sits at the midpoint between the advantages and limitations of saturation and inversion schemes [6].

Highly accurate $\mathrm{T} 1$ estimates can also be obtained with three-dimensional (3D) pulse sequences based on saturation recovery and developed to fully cover the left ventricle (LV). 3D SASHA combines a 2D SASHA-based pulse scheme with free-breathing imaging for 3D acquisition at 1.5T [11]. A more recent free-breathing 3D T1 mapping sequence provides $3 \mathrm{D}$ acquisition at $3 \mathrm{~T}$, based on a new pulse scheme that acquires substantially fewer T1-weighted images than 3D SASHA [12]. These 3D saturation recovery approaches offer higher SNR and good spatial coverage at the cost of longer acquisition times. They require navigator-triggered free-breathing and, therefore, rely on the respiratory navigation performance to achieve good image quality and acceptable acquisition time. Acquisition time is nevertheless in the range of minutes, even when denoising and optimization techniques are used $[13,14]$. Such long acquisition time compromises the feasibility of T1 mapping during contrast equilibrium and reduce clinical applicability. Furthermore, these 3D saturation recovery sequences acquire all T1-weighted images in the same RR interval of the saturation pulse, which compromises T1 estimation quality, particularly for long T1s and high heart rates [15].

Independent of magnetic preparation, most proposed T1 mapping techniques are acquired using SSFP readout techniques. At 3T, SSFP sequences have been associated with higher energy deposition and increased off-resonance artifacts [16]. Alternatives to SSFP sequences are 
spoiled sequences. These sequences use fast low-angle shot (FLASH) imaging readouts to avoid off-resonance artifacts and to eliminate transverse relaxation time (T2) dependence $[12,17,18]$. The main limitation of FLASH schemes is the low SNR compared with SSFP sequences.

In addition to the development of pulse sequences, new techniques have emerged to accelerate acquisition. Compressed sensing exploits the sparsity or compressibility of CMR images and accelerates acquisition by undersampling without significantly degrading images [19]. This technique has been successfully used to accelerate 3D cardiac imaging [20-22]. Compressed SENSE is built on compressed sensing and incorporates components of the parallel acquisition technique SENSE [23]. Studies showing the feasibility of compressed SENSE have recently been published for T1 calculation in the brain [24] and for cardiac cine imaging [25]. As expected, the method significantly reduced acquisition time, particularly in 3D acquisitions.

In this study, we propose and validate the 3D saturation recovery compressed SENSE rapid acquisition (3D SACORA) imaging sequence, a new 3D T1 spoiled saturation recovery mapping technique for acquisition of the entire $\mathrm{LV}$ in a single breath-hold at $3 \mathrm{~T}$. The proposed technique combines flexible saturation time sampling, compressed SENSE, and sharing of the saturation pulses between two readouts acquired at different $R R$ intervals. This approach aims to achieve good quality single breath-hold saturation recovery 3D T1 mapping and stability over a wide range of heart rates (HRs).

\section{Methods}

\section{Pulse sequence design}

3D SACORA consists of three distinct blocks (Fig. 1). The sequence begins with proton density (PD) images to avoid $\mathrm{T} 1$ effects from prior saturation pulses (first block). Full signal recovery is ensured by waiting a minimum of $6 \mathrm{~s}$ between the PD readouts $[12,26]$. The second block consists of images acquired at saturation time (TS) 1 and at TS1+RR interval (TS3). Finally, the third block of images is acquired at TS2 and at TS2 $+n$ RR intervals (TS4). T1 estimation is optimized not only by the PD images, but also by the selection of the acquired saturation time images [27]. TS1 is set to $250 \mathrm{~ms}$ to acquire the shortest possible saturation image, while maintaining an adequate SNR. For most heart rates, TS2 is set to $500 \mathrm{~ms}$ to allow a TS4 similar to the native cardiac T1 (1500 ms at 3T). For high heart rates that make a 500-ms TS2 unfeasible, the sequence automatically computes the longest possible TS2 according to the RR interval derived from the heart rate defined by the user in the acquisition protocol. In this way, TS1 and TS2 ensure consistent sampling at the low saturation time area of the T1 relaxation curve for both low and high heart rates. TS3 is always
Fig. 1 3D SACORA pulse sequence. The sequence performs two turbo field echo (TFE) shots for each 3D image: in the PD image block (top), the two TFEs are separated by a minimum $6 \mathrm{~s}$ magnetization recovery; two images are acquired at TS1 and TS3 (middle row); and two images are acquired at TS2 and TS4 (bottom row). TS1 $=250 \mathrm{~ms}$, TS2 $=500 \mathrm{~ms}$ (if allowed by the $\mathrm{HR}), \mathrm{TS} 3=\mathrm{TS} 1+\mathrm{RR}$ interval, $\mathrm{TS} 4=\mathrm{TS} 2+n \mathrm{RR}$ intervals $(n$ depending on HR)
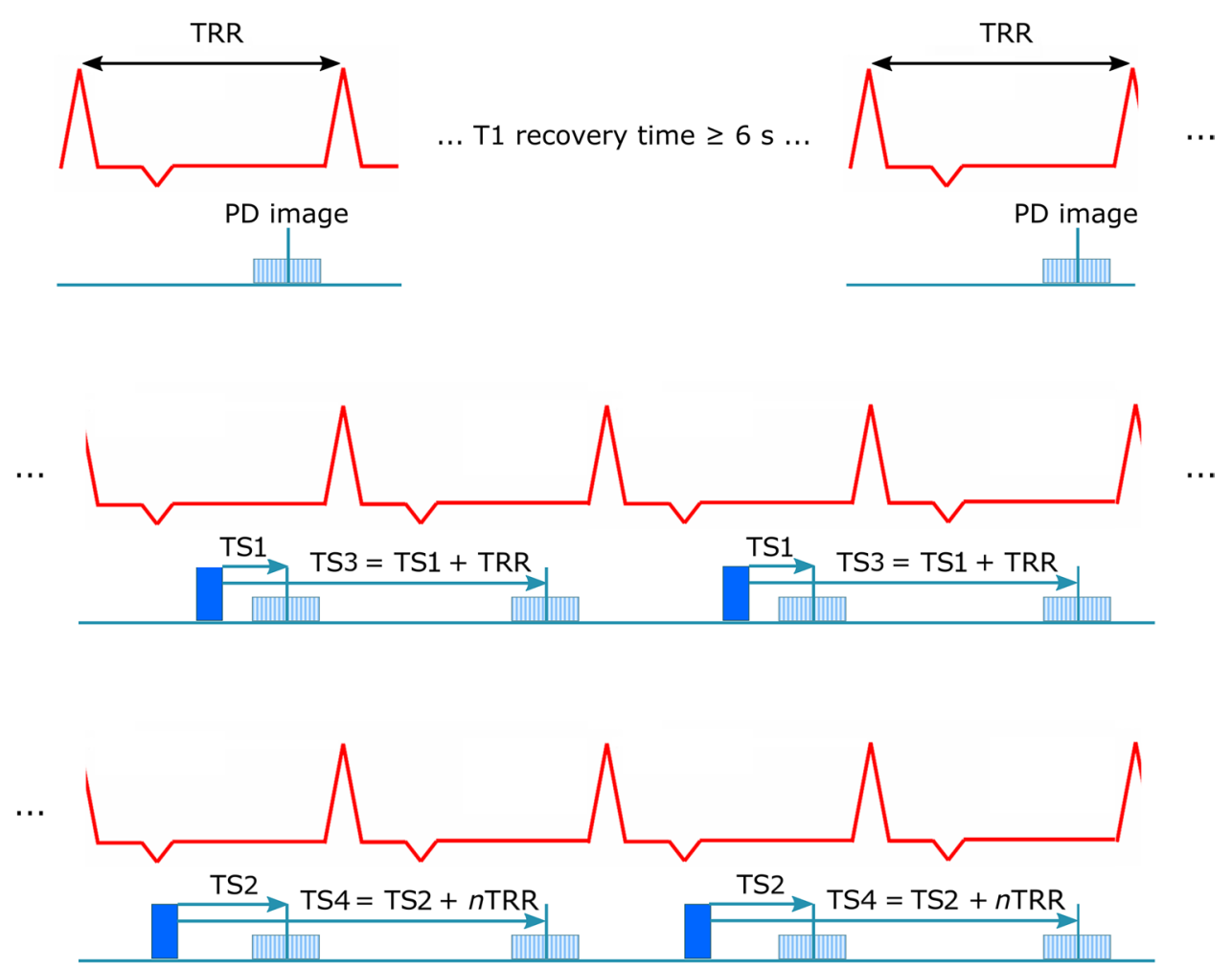
acquired at the RR interval immediately after TS1; whereas, TS4 is calculated to allow acquisition as close as possible to $1500 \mathrm{~ms}$; therefore, TS4 is acquired after $n$ recovery heart beats computed according to the heart rate. In this way, the design ensures sampling close to the $\mathrm{T} 1 \mathrm{~s}$ of interest and that TS3 and TS4 are unconstrained by high heart rates, as shown in Table $\mathrm{S} 1$.

3D SACORA uses a spoiled linear turbo field echo (T1-TFE) acquisition $\left(\mathrm{TR} / \mathrm{TE} / \mathrm{FA}=2.8 \mathrm{~ms} / 1.32 \mathrm{~ms} / 5^{\circ}\right)$. The $3 \mathrm{D}$ sequence covers a volume of $322 \times 322 \times 60 \mathrm{~mm}$ with an in-plane resolution of $2.0 \times 2.0 \mathrm{~mm}$ and a slice thickness of $10 \mathrm{~mm}$ reconstructed to $5 \mathrm{~mm}$ (12 slices). Composite radiofrequency $(\mathrm{RF})$ pulses are used to achieve homogeneous saturation across different $\mathrm{T} 1$ values $[28,29]$. Full image acquisition is accelerated using a k-space shutter and a spatial domain compressed SENSE factor of 4.5 to acquire a whole $3 \mathrm{D}$ volume in 2 independent TFE shots of echo train length of 76. Compressed SENSE technique solves an inverse problem with a sparsity constraint from data acquired using a balanced variable density incoherently undersampled k-space acquisition scheme with Poisson disc-style distribution. For reconstruction, the image is iteratively optimized according to the noise decorrelation, regularization, and coil sensitivities using a sparsity term based on wavelets transform [24, 30]. In this study, compressed SENSE was applied in the spatial domain in both phase-encoding directions with a fixed regularization parameter (maximum energy loss percentage equal to 30\%) to keep a good balance between data consistency, sparsity constraint and noise reduction. The effect of different regularization parameters/denoising levels is shown in Fig. S1.

\section{Image processing}

For 3D SACORA, T1 values are estimated by bounded Levenberg-Marquardt fitting using Bloch equations [5]. All sampling points are corrected according to the linear readout, and sampling points TS3 and TS4 are further corrected for the magnetization distortion caused by the readout effects of TS1 and TS2, respectively [31-33]. The T1 curves are fitted to a three-parameter (T1, $\alpha$ and $\mathrm{M} 0)$ relaxation model following the signal model described in Fig. S2. Fitting algorithm and T1 map generation are integrated in the scanner reconstruction pipeline; therefore, the parametric T1 maps for the entire 3D volume are available almost immediately after acquisition.

\section{Validation}

All studies were performed with a Philips Achieva 3T-Tx magnetic resonance imaging (MRI) scanner (Philips Healthcare, Best, the Netherlands) using a 32-channel cardiac coil.
The acquisition parameters and T1 maps generation methodology were optimized for in vivo acquisition and validated with phantom experiments.

\section{Phantom validation}

The precision and accuracy of 3D SACORA were assessed in phantom experiments. The phantom consisted of eight tubes filled with distilled water and different concentrations of an MR contrast agent (Dotarem, Guerbet, Paris, France) selected to obtain T1s spanning the range from $355 \mathrm{~ms}$ to $1871 \mathrm{~ms}$.

Reference T1 values were obtained using an inversion recovery spin-echo (IR-SE) sequence with a repetition time of $15 \mathrm{~s}$ and 15 inversion times ranging from 100 to $3500 \mathrm{~ms}$. Other sequence parameters were slice thickness $=10 \mathrm{~mm}$ and $\mathrm{TE}=18 \mathrm{~ms}$. Each image was acquired in approximately $11 \mathrm{~min}$.

To validate further 3D SACORA T1 estimation accuracy and precision, $\mathrm{T} 1$ values were also estimated using an inhouse version of 3D SASHA [11]. This 3D SASHA variant used a linear spoiled T1-TFE readout without any respiratory trigger for phantom and in vivo experiments. In addition to the PD image, eight equally spaced saturation time images were acquired, starting from a minimum saturation time of $120 \mathrm{~ms}$ to a maximum saturation time dependent of the heart rate- $709 \mathrm{~ms}$ in case of a heart rate of $60 \mathrm{bpm}$. PD recovery beats were computed automatically according to the heart rate so as to achieve a minimum $\mathrm{T} 1$ recovery time of $6 \mathrm{~s}$. Other protocol characteristics were TR/TE/FA $=2.8 \mathrm{~ms} / 1.3 \mathrm{~ms} / 12^{\circ}$; slice thickness $=10 \mathrm{~mm}$, reconstructed to $5 \mathrm{~mm}$ (12 slices); spatial domain compressed SENSE factor $=1.8$; in-plane resolution $=2 \times 2 \mathrm{~mm}$; Cartesian radial k-space filling; and echo train length $=30$. For a heart rate of $60 \mathrm{bpm}$, the acquisition time was approximately 3 minutes. The bounded three-parameter relaxation model-fitting algorithm and the T1 map generator were integrated in the scanner reconstruction pipeline.

T1 values (mean \pm standard deviation) were measured from manually drawn regions of interest (ROIs) in the generated T1 maps.

Passing-Bablok regression and Bland-Altman plots were used to assess correlation and agreement between the three sequences.

To explore the sensitivity of the proposed methodology to heart rate, we acquired images with 3D SACORA and 3D SASHA at simulated heart rates ranging from 50 to 120 $\mathrm{bpm}$. The estimated T1s were then compared against the T1 reference values obtained with IR-SE. As described before, both sequences automatically calculated the PD recovery beats, ensuring acquisition of fully recovered PD. Furthermore, the TS4 in 3D SACORA was automatically adjusted according to the heart rate to ensure acquisition within the 
range of T1 values of interest. The precision and accuracy of this approach were measured by the coefficient of variation:

$\mathrm{CV}=\frac{\mathrm{SD}}{\mathrm{M}}$

and relative error:

$\mathrm{RE}=\frac{M_{\text {proposedMethod }}-M_{\text {referenceMethod }}}{M_{\text {referenceMethod }}}$,

where $\mathrm{SD}$ is the standard deviation inside the ROI, $\mathrm{M}$ is the average value inside the ROI and reference method is the T1 map obtained from the IR-SE acquisition.

\section{In vivo validation}

Seven large white castrated male healthy pigs (mean weight, $40 \mathrm{~kg}$ ) were scanned with 3D SACORA and 3D SASHA. Mean heart rate during 3D SACORA pre-contrast acquisition was $73 \mathrm{bpm}$ (range, 55-83 bpm). In all pigs, images were acquired before and after administration of MR contrast agent. Scans were carried out under free-breathing conditions. No respiratory navigator approach was implemented because abdominal respiration in pigs produces little chest movement under free-breathing conditions.

The study protocol was approved by the local institutional Animal Research Committee and conducted in accordance with recommendations of the Guide for the Care and Use of Laboratory Animals.

Bulls-eye plots of $\mathrm{T} 1$ and coefficient of variation were generated from 3D SACORA and 3D SASHA data according to the American Heart Association (AHA) standard 17-segment model of the LV. For both techniques, the central segment was used to report blood values. The segment values used were mean values of the seven pigs. These plots were used to provide information about myocardium homogeneity and give a 3D perspective on the estimated $\mathrm{T} 1 \mathrm{~s}$.

For 3D SACORA and 3D SASHA, septal T1 values (mean \pm standard deviation) were measured from ROIs manually drawn on the septal myocardium. These measurements were used to compare the septal T1 values and coefficient of variation obtained by both sequences.

\section{Results}

\section{Phantom validation}

The Passing-Bablok regression plots presented in Fig. 2 show good correlation and no significant bias between methods. The 3D SACORA plot (Fig. 2a) has a slope of 0.99 (95\% confidence interval [CI] 0.98, 1.01) and an intercept of $12.90 \mathrm{~ms}(95 \% \mathrm{CI}-6.19,23.02 \mathrm{~ms})$; whereas, the 3D
SASHA plot (Fig. 2b) has a slope of 1.005 (95\%CI 0.997, $1.013)$ and an intercept of $-4.62 \mathrm{~ms}(95 \% \mathrm{Cl}-11.60,2.47$ $\mathrm{ms})$.

The Bland-Altman plots in Fig. 2 compare reference T1s with T1s estimated by 3D SACORA and 3D SASHA. These plots show good agreement between both methods and IR-SE. For 3D SACORA (Fig. 2c), the bias was -2.3 ms (95\% CI $-18.79,14.14$ ms). For 3D SASHA (Fig. 2d), the bias was $-0.45 \mathrm{~ms}(95 \% \mathrm{CI}-8.71,7.81 \mathrm{~ms})$.

The relative error at different heart rates ranged from -0.016 to 0.032 for 3D SACORA (mean, 0.008; standard deviation, 0.011); for 3D SASHA the range was -0.0095 to 0.039 (mean, 0.008; standard deviation, 0.01) (Fig. 3a, b). 3D SACORA showed less dependence on heart rate, particularly for pre-contrast cardiac $\mathrm{T} 1$ values at 3T; estimated T1 values with 3D SASHA tended to increase with higher heart rate; whereas, 3D SACORA T1s seemed to be relatively independent of heart rate. Regarding accuracy, both sequences performed equally well.

The coefficient of variation at different heart rates ranged from 0.013 to 0.054 for 3D SACORA (mean, 0.025; standard deviation, 0.011); for 3D SASHA, the range was 0.006-0.023 (mean, 0.011; standard deviation, 0.004) (Fig. 3c, d). Although precision was acceptable with both sequences, 3D SASHA performed slightly better, particularly on short T1s. The coefficient of variation appeared to be independent of heart rate in both sequences.

\section{In vivo validation}

Scans of the seven pigs were performed with 3D SACORA and 3D SASHA before and after MR contrast agent administration. 3D SACORA acquired the 3D T1 map in 15 heart beats (heart rate, $60 \mathrm{bpm}$ ). For both sequences, the final $\mathrm{T} 1$ parametric maps were obtained from the scanner shortly after acquisition. Reconstruction, fitting, and image generation took approximately 20 s.

Four images from the same animal for each sequence are shown in Fig. 4: two T1-weighted images, a PD image, and the corresponding T1 map. All T1-weighted images showed good contrast and quality. The TS1 image from 3D SACORA presented some noise, likely due to the short saturation time and relatively high compressed SENSE factor (4.5). No contrast was visible between myocardium and blood pool in the PD images, as expected. The T1 maps correctly represented the information contained in the T1-weighted images.

Bulls-eye plots were calculated for 3D SACORA and 3D SASHA according to the AHA LV model (Fig. 5). Bullseye plots of mean $\mathrm{T} 1$ values (Fig. 5a, b) showed acceptable homogeneity across the LV myocardium for both sequences. The mean T1 for the whole LV myocardium was $1480 \pm 33$ ms with 3D SACORA and $1539 \pm 54$ ms with 3D SASHA. For the blood pool, the mean T1 values were $2126 \pm 104$ 
(a)

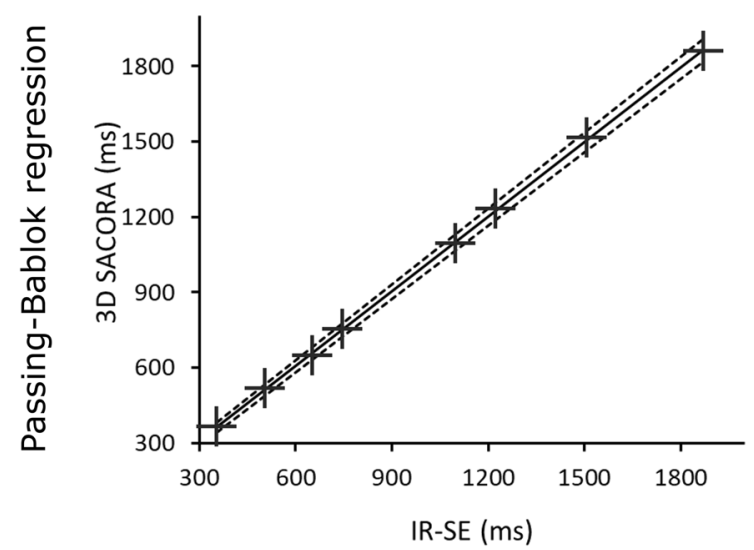

(c)

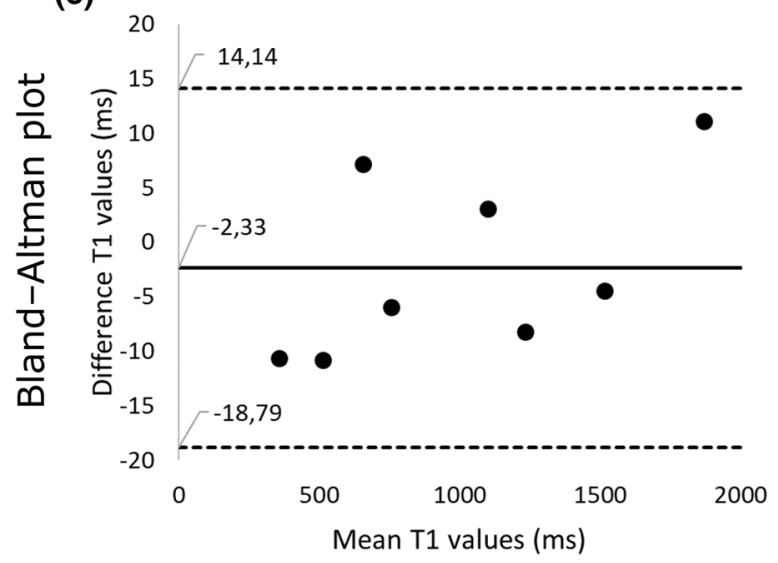

(b)

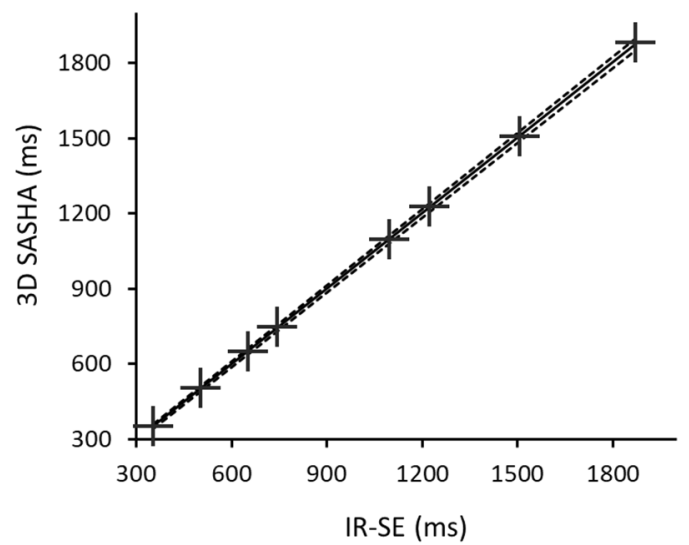

(d)

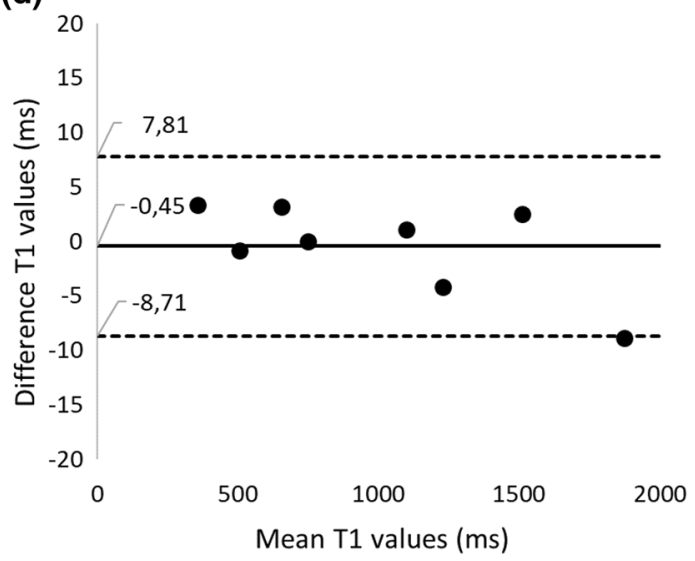

Fig. 2 3D SACORA and 3D SASHA results from phantom experiments validated against IR-SE. a, b Passing-Bablok regressions of 3D SACORA and 3D SASHA. c, d Bland-Altman plots of 3D SACORA and 3D SASHA

$\mathrm{ms}$ and $2306 \pm 93 \mathrm{~ms}$, respectively. The difference between the two sequences in mean whole LV myocardium T1 was mainly due to $\mathrm{T} 1$ measurements in the lateral and anterior segments. The bulls-eye plots for coefficient of variation (Fig. 5c, d) showed good precision in the measurement of $\mathrm{T} 1$ values in myocardium and blood for both sequences. The mean coefficients of variation in 3D SACORA were $0.029 \pm$ 0.005 in the whole $\mathrm{LV}$ myocardium and $0.033 \pm 0.006$ in the blood pool; the corresponding values for 3D SASHA were $0.030 \pm 0.008$ and $0.029 \pm 0.005$.

Septal T1s and coefficients of variation were measured with 3D SACORA and 3D SASHA in all seven pigs before and after administration of MR contrast agent (Fig. 6). Native and post-contrast T1 (Fig. 6a, b) did not differ between the two sequences, confirming good accuracy and precision. Mean septal native and post-contrast T1s measured with 3D SACORA were $1453 \pm 44 \mathrm{~ms}$ and $824 \pm 66$ $\mathrm{ms}$, respectively. For 3D SASHA, the mean septal native T1 was $1460 \pm 60 \mathrm{~ms}$ and the mean septal post-contrast $\mathrm{T} 1$ was $824 \pm 60 \mathrm{~ms}$. The coefficient of variation plots (Fig. 6c, d) showed acceptable precision for both sequences in all animals. The mean coefficient of variation for native septal T1 was $0.041 \pm 0.010$ for 3D SACORA and $0.039 \pm 0.010$ for 3D SASHA. The post-contrast values were $0.050 \pm 0.008$ and $0.041 \pm 0.008$, respectively.

Representative pre-contrast and post-contrast T1 maps of three slices (apex, middle, base) acquired with 3D SACORA and 3D SASHA in two pigs are shown in Fig. 7. 3D SACORA images showed good contrast, acceptable homogeneity, and were comparable to corresponding 3D SASHA images, despite the shorter acquisition time (15s vs. 188s, for a heart rate of $60 \mathrm{bpm}$ ).

\section{Discussion}

3D SACORA (3D saturation recovery compressed SENSE rapid acquisition) was developed as a new 3D T1 mapping sequence to speed up T1 mapping acquisition of the whole 

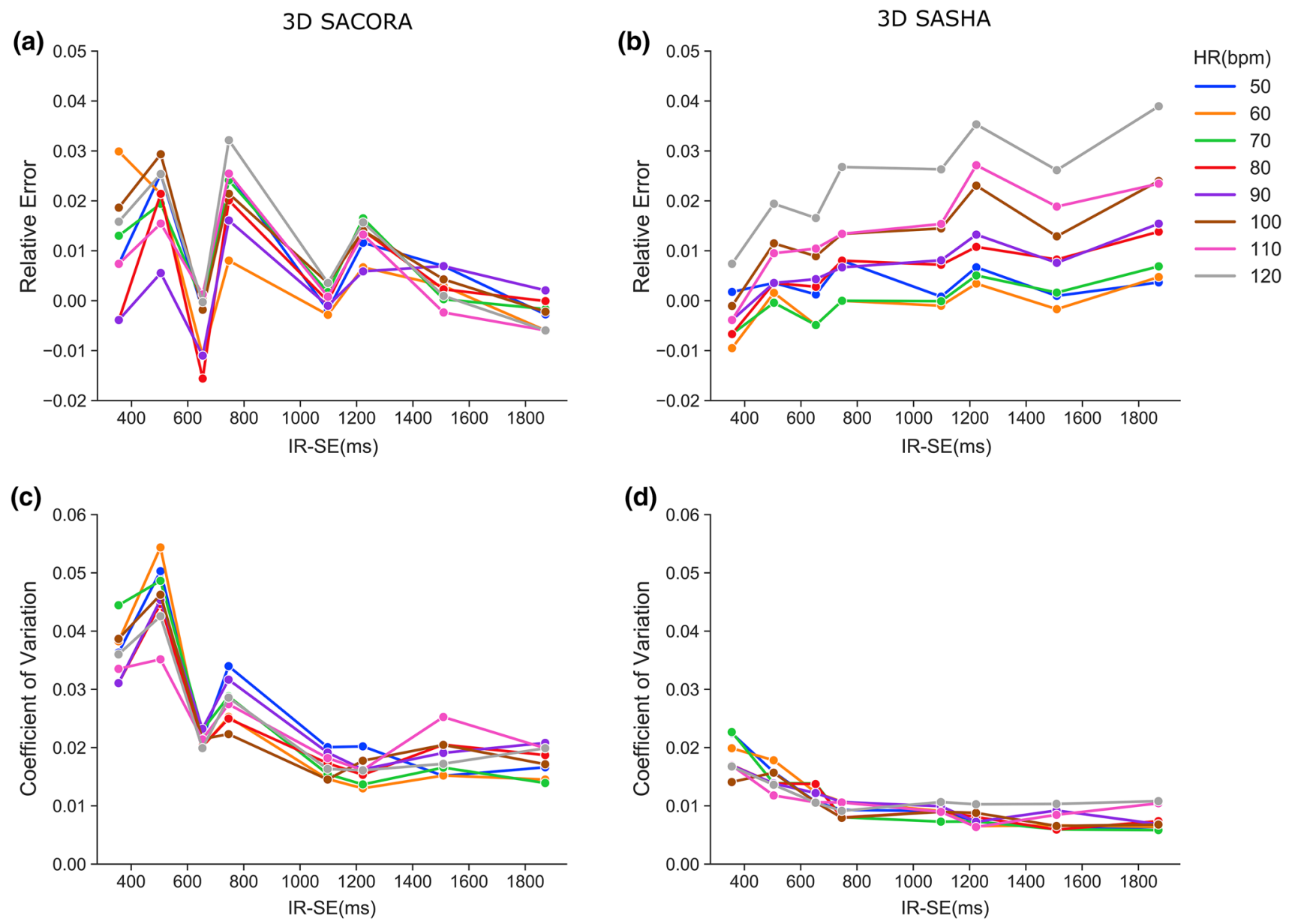

Fig. 3 Phantom experiments assessing the HR dependency of 3D SACORA and 3D SASHA. a, b Relative error of 3D SACORA and 3D SASHA at different HRs. c, $\mathbf{d}$ Coefficient of variation of 3D SACORA and 3D SASHA at different HRs

Fig. 4 Representative images acquired from pig 6 with 3D SACORA and 3D SASHA (two T1-weighted images and a PD image) and the corresponding T1 maps
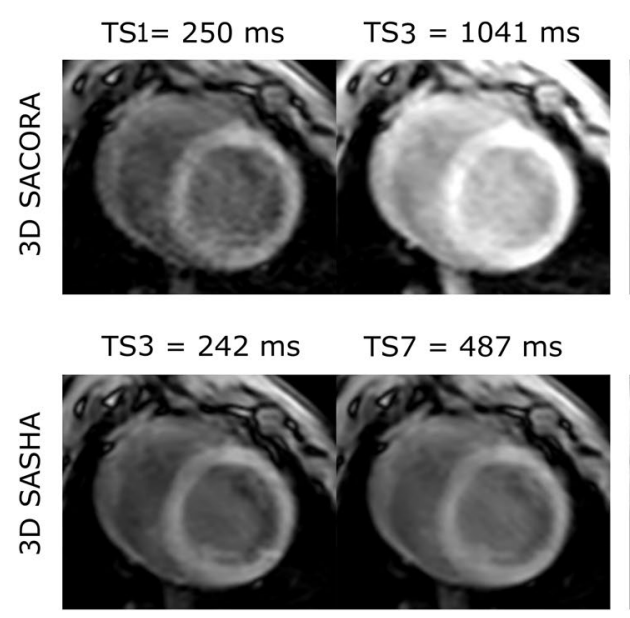

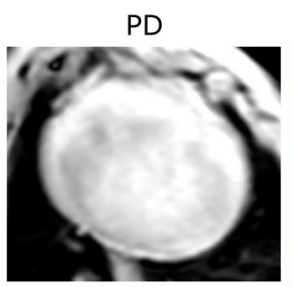

PD

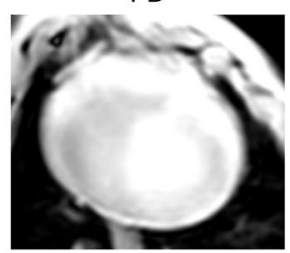

T1 map

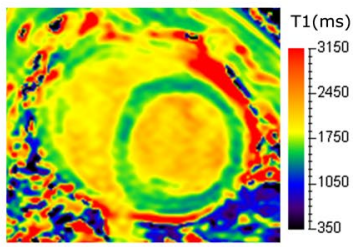

T1 map

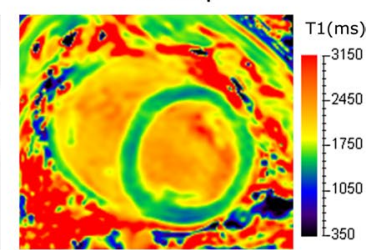

heart. The proposed sequence was validated in phantoms against the gold standard technique (IR-SE) and in vivo against the previously proposed sequence 3D SASHA.
3D SACORA successfully acquired a whole-heart 3D T1 map in a single breath-hold at 3T, estimating T1 values in agreement with those obtained with the IR-SE and 3D SASHA sequences. Thus, 3D SACORA's use of 5 saturation 

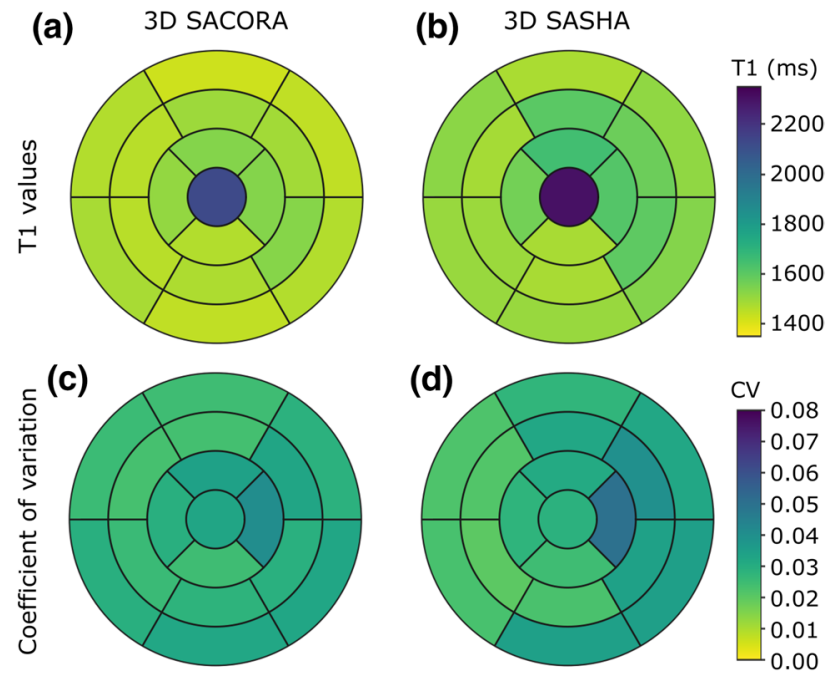

Fig. 5 Bulls-eye plots of T1 and coefficient of variation (CV) for the whole left-ventricle myocardium with 3D SACORA and 3D SASHA. The central segment corresponds to the blood. a, b Bulls-eye plots of mean $\mathrm{T} 1$ values for both sequences. $\mathbf{c}, \mathbf{d}$ Bulls-eye plots of mean CV for both sequences

times for T1 fitting as well as k-space under-sampling via compressed SENSE enabled very short acquisition times $(15 \mathrm{~s}$, for a heart rate of $60 \mathrm{bpm})$ without significantly compromising $\mathrm{T} 1$ estimation accuracy or image quality.

$3 \mathrm{D}$ saturation recovery $\mathrm{T} 1$ mapping sequences have been developed recently because they do not require full longitudinal magnetization recovery and produce highly accurate $\mathrm{T} 1$ values. Acquisition times with these $3 \mathrm{D}$ sequences are, however, much longer than $20 \mathrm{~s}$, and scans, therefore, cannot be performed under single breath-hold conditions. 3D SACORA was optimized to keep scan time shorter than $20 \mathrm{~s}$ without compromising T1 estimation accuracy, image quality, or LV coverage. Conditions established for this optimization included (i) allowing enough time for the PD to achieve full magnetization recovery between readouts, (ii) limiting the number of turbo field echo shots as much as possible, (iii) optimizing trade-off between readout length and the compressed SENSE factor, (iv) acquiring T1-weighted images with enough SNR for proper application of compressed SENSE, and (v) acquiring a T1-weighted image with a saturation time as close as possible to the native cardiac $\mathrm{T} 1$ values at $3 \mathrm{~T}$.

3D SACORA is able to acquire a 3D T1 map in 15 heart beats (heart rate, $60 \mathrm{bpm}$ ) at 3T. The main constraint for acquiring $\mathrm{T} 1$ maps with a short scan time at $3 \mathrm{~T}$ is the recovery beats required by the proton density. To mitigate this, $3 \mathrm{D}$ SACORA was designed to have just two readout shots. This was achieved by combining a shot length of $\sim 210 \mathrm{~ms}$ with a compressed SENSE factor of 4.5, providing good image quality without major deterioration or blurring. A compressed SENSE factor of 4.5 guarantees good T1 estimation accuracy and precision, with comparable results to lower compressed SENSE factors, as shown in Fig. S3. A shot length of $\sim 210 \mathrm{~ms}$ is similar to the conventional 2D MOLLI shot length of $\sim 190 \mathrm{~ms}$ [4] and shorter than the diastolic time length at very high heart rates $(250 \pm 59 \mathrm{~ms}$ at $128 \pm 22$ bpm) [34]. The PD readouts were separated by $6 \mathrm{~s}$ to guarantee full magnetization recovery. 3D SACORA acquires two T1-weighted images with long saturation times to enhance fitting quality for relevant cardiac $\mathrm{T} 1$ values at $3 \mathrm{~T}$. In addition, these images are ideal for applying compressed SENSE due to their high SNR; whereas, T1-weighted images with shorter saturation times tend to be noisier, as shown in Fig. 4.

3D SACORA and our own implementation of 3D SASHA were validated in phantom experiments. 3D SASHA is the reference sequence for in vivo experiments in this study and has an acquisition time roughly 13 times longer than 3D SACORA. The phantom results show that 3D SACORA and 3D SASHA acquired 3D T1 maps with high accuracy and precision and in good agreement with IR-SE measurements. However, slight differences were found between 3D SACORA and 3D SASHA in specific cases (Fig. 3). First, 3D SASHA is more precise than 3D SACORA for short T1s, due to the denser sampling of short saturation time T1-weighted images present in 3D SASHA. Second, 3D SACORA T1 estimation is, by design, less heart-rate sensitive than 3D SASHA. Heart-rate sensitivity in 3D SASHA acquisition is due to its lack of long saturation images, which can undermine $\mathrm{T} 1$ estimation quality, especially at high heart rates. Thus, the 3D SACORA sampling strategy was validated over a wide range of heart rates $(50 \mathrm{bpm}-120$ $\mathrm{bpm})$. For heart rates outside this range, the sequence keeps acquiring saturation times at the low saturation time area of the T1 relaxation curve and close to the T1s of interest, as shown in Table S1. This sampling strategy of the saturation times makes 3D SACORA robust for a very wide range of heart rates.

The pig heart is an established model in cardiology due to its similarity to the human heart $[35,36]$. In this study, we acquire in vivo data before and after contrast administration. The image quality in 3D SACORA was close to that obtained with 3D SASHA despite the much shorter acquisition time. All septal T1 measurements were similar in the two sequences (Fig. 6a, b), despite the differences in sequence design and protocol. Furthermore, the mean septal pre-contrast T1 of $1453 \mathrm{~ms}$ estimated by 3D SACORA is in good agreement with published saturation recovery $\mathrm{T} 1$ measurements in pigs at 3T [29]. There was a slight discrepancy between 3D SACORA and 3D SASHA in T1s measured in the lateral and anterior segments of the myocardium (Fig. 5a, b), probably caused by movement artifacts, which were more frequent in 3D SASHA due to the longer acquisition time. 
(a)

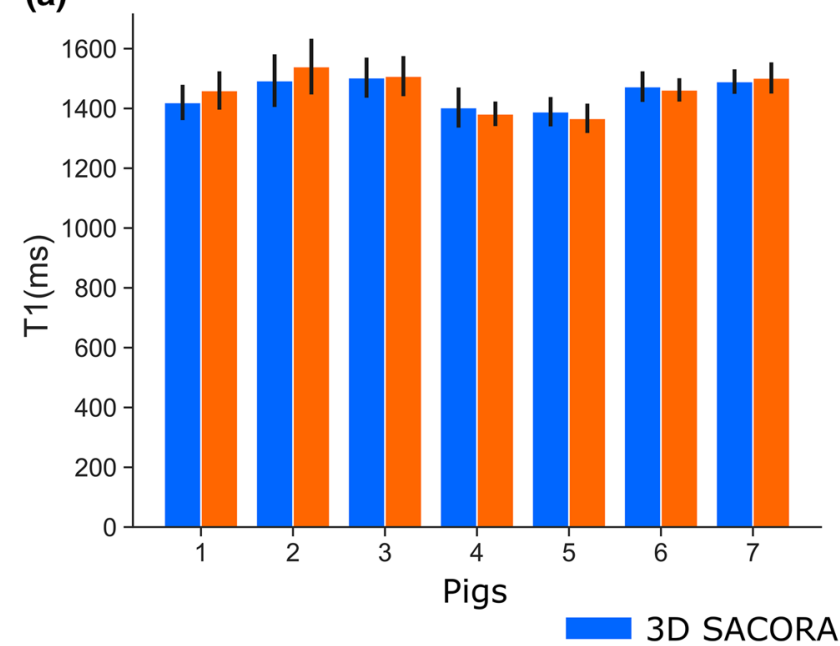

(c)

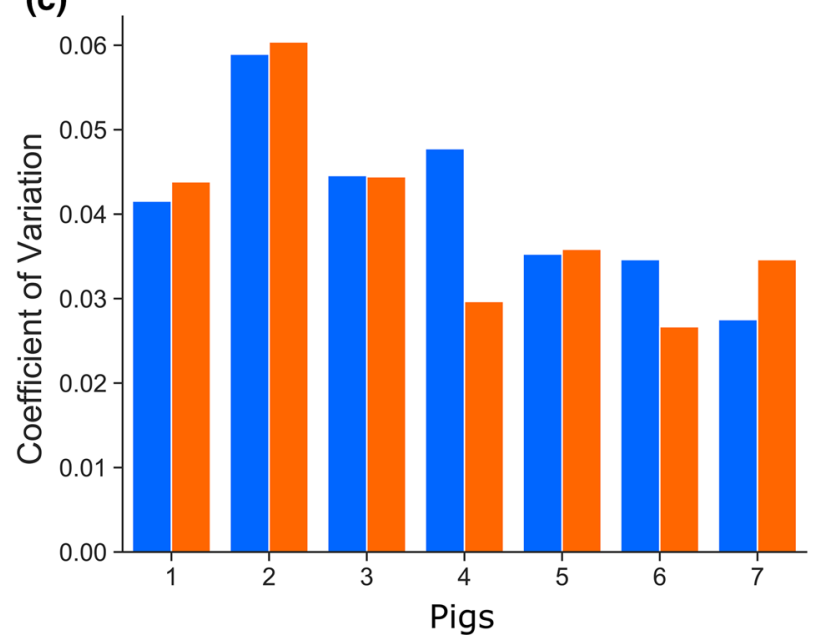

Fig. 6 Septal T1 measurements (pre-contrast and post-contrast) obtained from all pigs with 3D SACORA and 3D SASHA. a Precontrast septal $\mathrm{T} 1$ and standard deviation for both sequences. b Post-contrast septal T1 and standard deviation for both sequences.

In pigs, cardiac acquisitions performed in free-breathing are free of major respiratory artifacts $[36,37]$. This was crucial for the successful comparison of 3D SACORA with 3D SASHA in a model without respiratory motion compensation (e.g., respiratory navigator [12] or motion correction [38]) or breath-hold acquisition. Nevertheless, the results might be improved by taking appropriate measures to minimize respiratory artifacts. For example, T1 map quality could be improved by reducing respiration-induced motion using registration approaches such as non-rigid image registration [39].

In this study, we used an in-house version of 3D SASHA for $3 \mathrm{~T}$ [11]. As the reference sequence for the in vivo

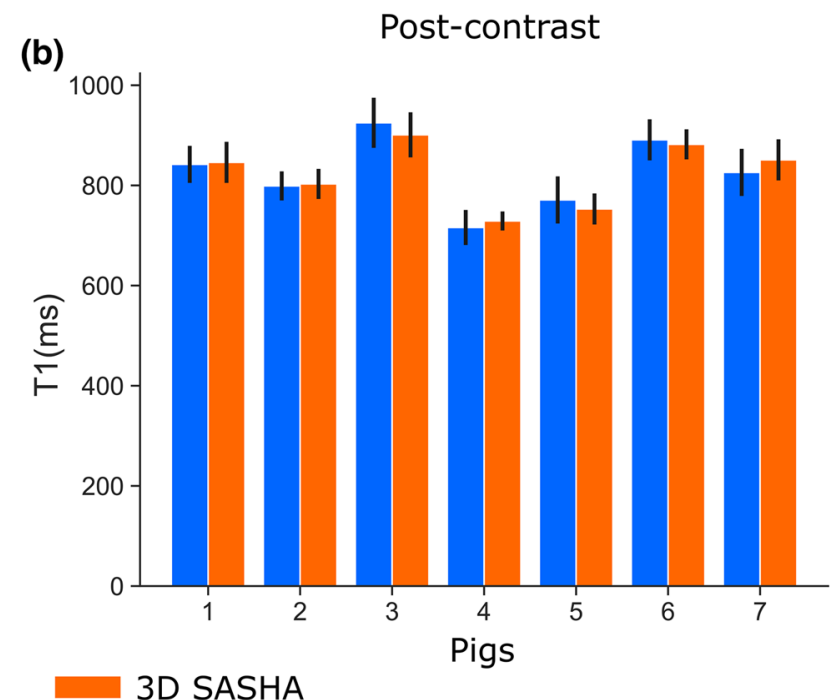

(d)

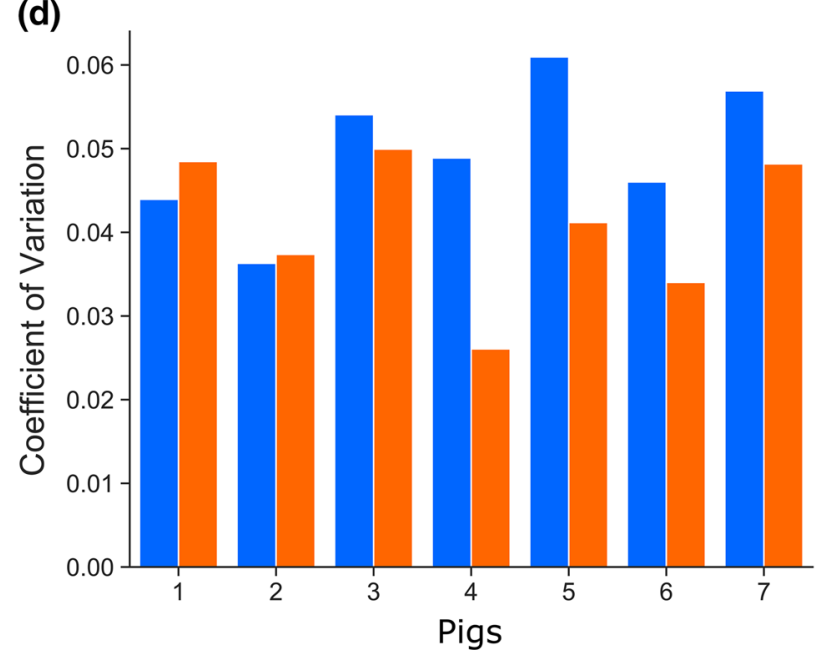

c Septal coefficient of variation of pre-contrast acquisitions for both sequences. d Septal coefficient of variation of post-contrast acquisitions for both sequences

experiments, the sampling strategy of the saturation times was similar to conventional 3D SASHA to acquire gold standard in vivo T1 values. In addition, 3D SASHA was implemented with a shorter shot length than 3D SACORA, minimizing the effect of cardiac motion and reducing partial volume averaging, which are especially relevant for high heart rates. This sequence was successfully validated against IR-SE with phantom experiments, and the in vivo results were in good agreement with published saturation recovery T1 measurements at 3T [12, 29].

Despite the good performance of 3D SACORA in postcontrast imaging, the sequence was primarily designed for pre-contrast imaging. Although post-contrast T1 values 


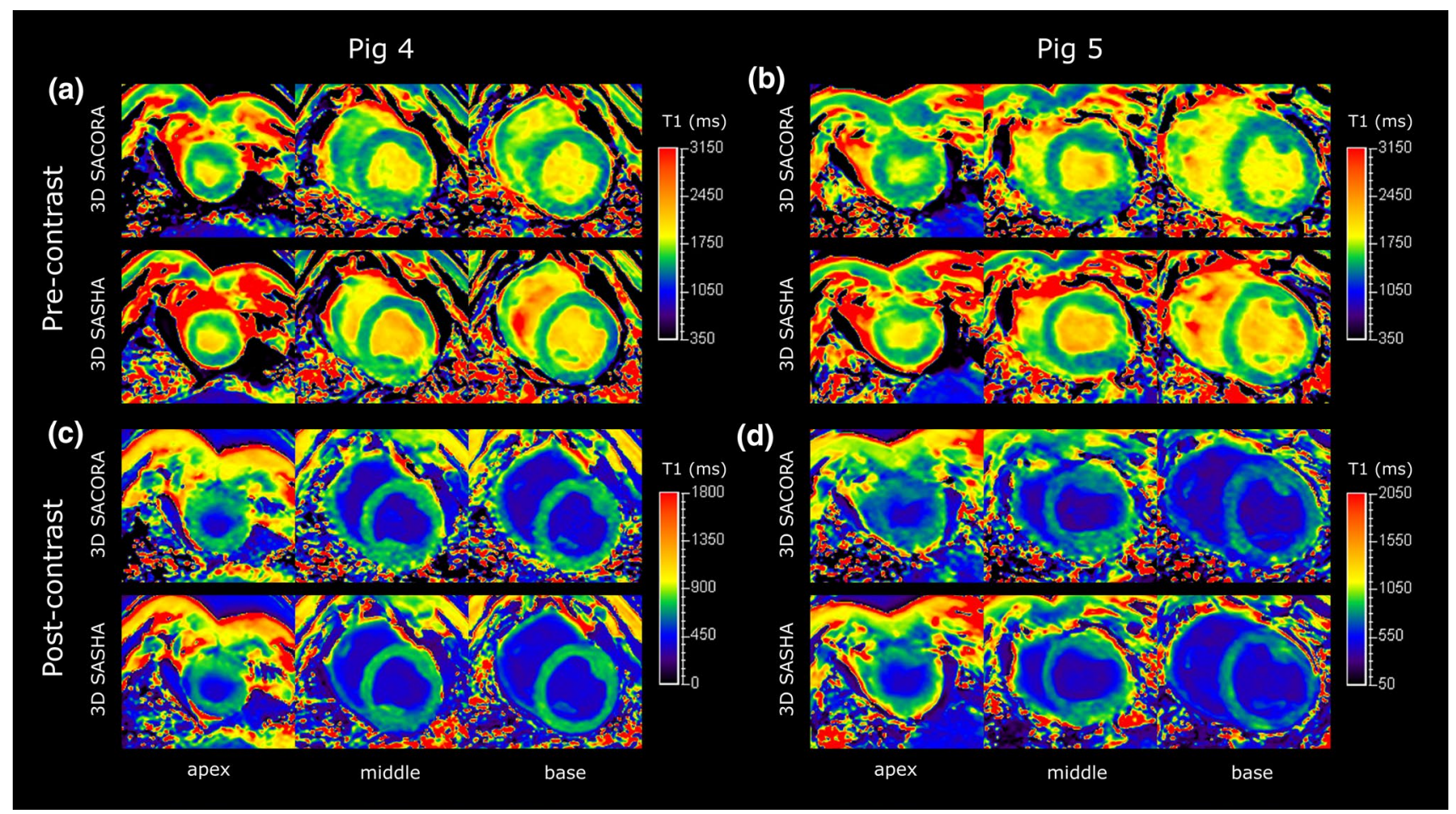

Fig. 7 Pre-contrast and post-contrast T1 maps of three slices (apex, middle, base) acquired with 3D SACORA and 3D SASHA from pigs 4 and 5. a, b Pre-contrast images from pigs 4 and 5. c, d Post-contrast images from pigs 4 and 5

obtained with the proposed technique do not differ significantly from those obtained with 3D SASHA, the accuracy and precision of short $\mathrm{T} 1$ could be improved by increasing the number of short saturation images. The additional acquisition time could be compensated by decreasing the number of PD recovery beats.

One of the main limitations of this feasibility study is the lack of in vivo human data. Nevertheless, pig model is a well-stablished model for cardiac research and in vivo pig acquisitions can be performed under free-breathing conditions without significantly decreasing image quality [35-37]. Additionally, feasibility studies of new cardiac acquisitions have been successfully performed on pigs [40-43]. A clinical study will be required to evaluate the performance of 3D SACORA (single breath-hold of 15s, for a heart rate of $60 \mathrm{bpm}$ ) in patients with ischemic and non-ischemic cardiomyopathies.

In conclusion, the proposed 3D SACORA sequence acquired pre-contrast and post-contrast $\mathrm{T} 1$ maps of the whole heart with good accuracy, precision, and image quality for LV analysis at 3T. The sequence was optimized for speed and can acquire a 3D T1map in 15 heart beats for a heart rate of $60 \mathrm{bpm}$.
Acknowledgements TFdS has received funding from the European Union Horizon 2020 research and innovation programme under the Marie Sklodowska-Curie grant agreement N722427. CGA is a P-FIS fellow (Instituto de Salud Carlos III). This study was partially supported by the Comunidad de Madrid (S2017/BMD-3867 RENIM$\mathrm{CM}$ ) and cofunded with European structural and investment funds. The CNIC is supported by the Instituto de Salud Carlos III (ISCIII), the Ministerio de Ciencia, Innovación y Universidades (MCNU) and the Pro CNIC Foundation, and is a Severo Ochoa Center of Excellence (SEV-2015-0505). Simon Bartlett (CNIC) provided English language editing. This study formed part of a Master Research Agreement between the CNIC and Philips Healthcare.

Author contributions TFdS, CGA, PM, GJLM, MD, VF, BI and JSG provided key ideas to the proposed methodology. TFdS and JSG developed the pulse sequences and map generation methodologies. TFdS, CGA, PM, GJLM and JSG acquired the data. TFdS, MD, VF, BI and JSG collaborated in the study design and data analysis. TFdS, CGA, PM, GJLM, MD, VF, BI and JSG collaborated in manuscript drafting and critical comments to the analysis of results. All authors read and approved the final manuscript.

Funding TFdS has received funding from the European Union Horizon 2020 research and innovation programme under the Marie SklodowskaCurie grant agreement N722427. CGA is a P-FIS fellow (Instituto de Salud Carlos III). This study was partially supported by the Comunidad de Madrid (S2017/BMD-3867 RENIM-CM) and cofunded with European structural and investment funds. The CNIC is supported by the Instituto de Salud Carlos III (ISCIII), the Ministerio de Ciencia, Innovación y Universidades (MCNU) and the Pro CNIC Foundation, and is a Severo Ochoa Center of Excellence (SEV-2015-0505). 


\section{Compliance with ethical standards}

Conflict of interest TFdS, PM and JSG are Philips employees.

Ethical standards The project was approved by the Comité de Ética de la Investigación y el Bienestar Animal del Instituto de Salud Carlos III.

Consent for publication Not applicable

Data and/or Code availability The data and code used and/or analyzed during the current study are available from the corresponding author on reasonable request.

Open Access This article is licensed under a Creative Commons Attribution 4.0 International License, which permits use, sharing, adaptation, distribution and reproduction in any medium or format, as long as you give appropriate credit to the original author(s) and the source, provide a link to the Creative Commons licence, and indicate if changes were made. The images or other third party material in this article are included in the article's Creative Commons licence, unless indicated otherwise in a credit line to the material. If material is not included in the article's Creative Commons licence and your intended use is not permitted by statutory regulation or exceeds the permitted use, you will need to obtain permission directly from the copyright holder. To view a copy of this licence, visit http://creativecommons.org/licenses/by/4.0/.

\section{References}

1. Haaf P, Garg P, Messroghli DR, Broadbent DA, Greenwood JP, Plein S (2016) Cardiac T1 Mapping and Extracellular Volume (ECV) in clinical practice: a comprehensive review. J Cardiovasc Magn Reson 18:89

2. Messroghli DR, Moon JC, Ferreira VM, Grosse-Wortmann L, He T, Kellman P, Mascherbauer J, Nezafat R, Salerno M, Schelbert EB, Taylor AJ, Thompson R, Ugander M, van Heeswijk RB, Friedrich MG (2017) Clinical recommendations for cardiovascular magnetic resonance mapping of $\mathrm{T} 1, \mathrm{~T} 2, \mathrm{~T} 2 *$ and extracellular volume: A consensus statement by the Society for Cardiovascular Magnetic Resonance (SCMR) endorsed by the European Association for Cardiovascular Imaging (EACVI). J Cardiovasc Magn Reson 19:75. https://doi.org/10.1186/s12968-017-0389-8

3. Ibanez B, Aletras AH, Arai AE, Arheden H, Bax J, Berry C, Bucciarelli-Ducci C, Croisille P, Dall' Armellina E, Dharmakumar R, Eitel I, Fernández-Jiménez R, Friedrich MG, García-Dorado D, Hausenloy DJ, Kim RJ, Kozerke S, Kramer CM, Salerno M, SánchezGonzález J, Sanz J, Fuster V (2019) Cardiac MRI Endpoints in Myocardial Infarction Experimental and Clinical Trials: JACC Scientific Expert Panel. J Am Coll Cardiol 74:238-256

4. Messroghli DR, Radjenovic A, Kozerke S, Higgins DM, Sivananthan MU, Ridgway JP (2004) Modified Look-Locker inversion recovery (MOLLI) for high-resolution T1 mapping of the heart. Magn Reson Med 52:141-146

5. Chow K, Flewitt JA, Green JD, Pagano JJ, Friedrich MG, Thompson RB (2014) Saturation recovery single-shot acquisition (SASHA) for myocardial T1 mapping. Magn Reson Med 71:2082-2095

6. Weingärtner S, Akçakaya M, Basha T, Kissinger KV, Goddu B, Berg S, Manning WJ, Nezafat R (2014) Combined saturation/ inversion recovery sequences for improved evaluation of scar and diffuse fibrosis in patients with arrhythmia or heart rate variability. Magn Reson Med 71:1024-1034
7. Cameron D, Vassiliou VS, Higgins DM, Gatehouse PD (2018) Towards accurate and precise T 1 and extracellular volume mapping in the myocardium: a guide to current pitfalls and their solutions. Magn Reson Mater Phy 31:143-163

8. Kellman P, Hansen MS (2014) T1-mapping in the heart: accuracy and precision. J Cardiovasc Magn Reson 16:2

9. Piechnik SK, Neubauer S, Ferreira VM (2018) State-of-the-art review: stress T1 mapping-technical considerations, pitfalls and emerging clinical applications. Magn Reson Mater Phy $31: 131-141$

10. Song T, Stainsby JA, Ho VB, Hood MN, Slavin GS (2012) Flexible cardiac T1 mapping using a modified look-locker acquisition with saturation recovery. Magn Reson Med 67:622-627

11. Nordio G, Henningsson M, Chiribiri A, Villa ADM, Schneider T, Botnar RM (2017) 3D myocardial T 1 mapping using saturation recovery. J Magn Reson Imaging 46:218-227

12. Guo R, Chen Z, Wang Y, Herzka DA, Luo J, Ding H (2018) Threedimensional free breathing whole heart cardiovascular magnetic resonance T1 mapping at 3 T. J Cardiovasc Magn Reson 20:64. https://doi.org/10.1186/s12968-018-0487-2

13. Nordio G, Bustin A, Henningsson M, Rashid I, Chiribiri A, Ismail T, Odille F, Prieto C, Botnar RM (2018) 3D SASHA myocardial T1 mapping with high accuracy and improved precision. Magn Reson Mater Phy 32:281-289

14. Nordio G, Schneider T, Cruz G, Correia T, Bustin A, Prieto C, Botnar RM, Henningsson M (2019) Whole-heart T 1 mapping using a 2D fat image navigator for respiratory motion compensation. Magn Reson Med 83:178-187

15. Stainsby JA, Slavin GS (2014) Comparing the accuracy and precision of SMART1Map, SASHA and MOLLI. J Cardiovasc Magn Reson 16:P11

16. Jogiya R, Schuster A, Zaman A, Motwani M, Kouwenhoven M, Nagel E, Kozerke S, Plein S (2014) Three-dimensional balanced steady state free precession myocardial perfusion cardiovascular magnetic resonance at 3T using dual-source parallel RF transmission: initial experience. J Cardiovasc Magn Reson 16:90

17. Gensler D, Mörchel P, Fidler F, Ritter O, Quick HH, Ladd ME, Bauer WR, Ertl G, Jakob PM, Nordbeck P (2015) Myocardial T1: Quantification by Using an ECG-triggered Radial SingleShot Inversion-Recovery MR Imaging Sequence. Radiology 274:879-887

18. Shao J, Rapacchi S, Nguyen K-L, Hu P (2016) Myocardial T1 Mapping at 3.0T Using an Inversion Recovery Spoiled Gradient Echo Readout and Bloch Equation Simulation with Slice Profile Correction (BLESSPC) T1 Estimation Algorithm. J Magn Reson Imaging JMRI 43:414-425

19. Lustig M, Donoho D, Pauly JM (2007) Sparse MRI: The application of compressed sensing for rapid MR imaging. Magn Reson Med 58:1182-1195

20. Liu J, Feng L, Shen H-W, Zhu C, Wang Y, Mukai K, Brooks GC, Ordovas K, Saloner D (2017) Highly-accelerated self-gated freebreathing 3D cardiac cine MRI: validation in assessment of left ventricular function. Magn Reson Mater Phy 30:337-346

21. Weingärtner S, Akçakaya M, Roujol S, Basha T, Tschabrunn C, Berg S, Anter E, Nezafat R (2015) Free-breathing combined three-dimensional phase sensitive late gadolinium enhancement and $\mathrm{T} 1$ mapping for myocardial tissue characterization: Integrated Sequence for 3D LGE Imaging and 3D T 1 Mapping. Magn Reson Med 74:1032-1041

22. Wetzl J, Schmidt M, Pontana F, Longère B, Lugauer F, Maier A, Hornegger J, Forman C (2018) Single-breath-hold 3-D CINE imaging of the left ventricle using Cartesian sampling. Magn Reson Mater Phy 31:19-31

23. Pruessmann KP, Weiger M, Scheidegger MB, Boesiger P (1999) SENSE: Sensitivity encoding for fast MRI. Magn Reson Med 42:952-962 
24. Vranic JE, Cross NM, Wang Y, Hippe DS, de Weerdt E, MossaBasha M (2019) Compressed Sensing-Sensitivity Encoding (CSSENSE) Accelerated Brain Imaging: Reduced Scan Time without Reduced Image Quality. Am J Neuroradiol 40:92-98

25. Ma Y, Hou Y, Ma Q, Wang X, Sui S, Wang B (2019) Compressed SENSE single-breath-hold and free-breathing cine imaging for accelerated clinical evaluation of the left ventricle. Clin Radiol 74(4):325.e9-325.e17. https://doi.org/10.1016/j.crad.2018.12.012

26. Guo R, Chen Z, Herzka DA, Luo J, Ding H (2019) A three-dimensional free-breathing sequence for simultaneous myocardial $\mathrm{T} 1$ and T 2 mapping. Magn Reson Med 81:1031-1043

27. Akçakaya M, Weingärtner S, Roujol S, Nezafat R (2015) On the Selection of Sampling Points for Myocardial T1 Mapping. Magn Reson Med 73:1741-1753

28. Morita K, Oda S, Utsunomiya D, Nakaura T, Matsubara T, Goto M, Okuaki T, Yuki H, Nagayama Y, Kidoh M, Hirata K, Iyama Y, Taguchi N, Hatemura M, Hashida M, Yamashita Y (2017) Saturation Recovery Myocardial T1 Mapping with a Composite Radiofrequency Pulse on a 3T MR Imaging System. Magn Reson Med Sci 17:35-41

29. Chow K, Kellman P, Spottiswoode BS, Nielles-Vallespin S, Arai AE, Salerno M, Thompson RB (2015) Saturation pulse design for quantitative myocardial T1 mapping. J Cardiovasc Magn Reson 17:84. https://doi.org/10.1186/s12968-015-0187-0

30. Zibulevsky M, Elad M (2010) L1-L2 Optimization in Signal and Image Processing. IEEE Signal Process Mag 27:76-88

31. Busse RF, Riederer SJ (2001) Steady-state preparation for spoiled gradient echo imaging. Magn Reson Med 45:653-661

32. Warntjes MJ, Kihlberg J, Engvall J (2010) Rapid T1 quantification based on 3D phase sensitive inversion recovery. BMC Med Imaging 10:19

33. Li W, Griswold M, Yu X (2010) Rapid T1 Mapping of Mouse Myocardium with Saturation Recovery Look-Locker Method. Magn Reson Med 64:1296-1303

34. Bombardini T, Gemignani V, Bianchini E, Venneri L, Petersen C, Pasanisi E, Pratali L, Alonso-Rodriguez D, Pianelli M, Faita F, Giannoni M, Arpesella G, Picano E (2008) Diastolic time frequency relation in the stress echo lab: filling timing and flow at different heart rates. Cardiovasc Ultrasound 6:15

35. García-Álvarez A, García-Lunar I, Pereda D, Fernández-Jimenez R, Sánchez-González J, Mirelis JG, Nuño-Ayala M, SánchezQuintana D, Fernández-Friera L, García-Ruiz JM, Pizarro G, Agüero J, Campelos P, Castellá M, Sabaté M, Fuster V, Sanz J, Ibañez B (2015) Association of Myocardial T1-Mapping CMR With Hemodynamics and RV Performance in Pulmonary Hypertension. JACC Cardiovasc Imaging 8:76-82

36. Galán-Arriola C, Lobo M, Vílchez-Tschischke JP, López GJ, de Molina-Iracheta A, Pérez-Martínez C, Agüero J, FernándezJiménez R, Martín-García A, Oliver E, Villena-Gutierrez R,
Pizarro G, Sánchez PL, Fuster V, Sánchez-González J, Ibanez B (2019) Serial Magnetic Resonance Imaging to Identify Early Stages of Anthracycline-Induced Cardiotoxicity. J Am Coll Cardiol 73:779-791

37. Rodrigo Fernández-Jiménez, Carlos Galán-Arriola, Javier Sánchez-González, Jaume Agüero, López-Martín Gonzalo J, Sandra Gomez-Talavera, Jaime Garcia-Prieto, Austin Benn, Antonio Molina-Iracheta, Manuel Barreiro-Pérez, Ana Martin-García, Inés García-Lunar, Gonzalo Pizarro, Javier Sanz, Sánchez Pedro L, Valentin Fuster, Borja Ibanez (2017) Effect of Ischemia Duration and Protective Interventions on the Temporal Dynamics of Tissue Composition After Myocardial Infarction. Circ Res 121:439-450

38. Qi H, Bustin A, Cruz G, Jaubert O, Chen H, Botnar RM, Prieto C (2019) Free-running simultaneous myocardial T1/T2 mapping and cine imaging with 3D whole-heart coverage and isotropic spatial resolution. Magn Reson Imaging 63:159-169

39. Roujol S, Foppa M, Weingartner S, Manning WJ, Nezafat R (2015) Adaptive Registration of Varying Contrast-Weighted Images for Improved Tissue Characterization (ARCTIC): Application to T1 Mapping. Magn Reson Med 73:1469-1482

40. Wang S-C, Patel AR, Tanaka A, Wang H, Ota T, Lang RM, Carroll TJ, Kawaji K (2017) A novel profile/view ordering with a non-convex star shutter for high-resolution $3 \mathrm{D}$ volumetric $\mathrm{T} 1$ mapping under multiple breath-holds: Novel Profile Ordering for 3D T 1 Mapping. Magn Reson Med 77:2215-2224

41. Kawaji K, Tanaka A, Patel MB, Wang H, Maffessanti F, Ota T, Patel AR (2017) 3D late gadolinium enhanced cardiovascular MR with CENTRA-PLUS profile/view ordering: Feasibility of right ventricular myocardial damage assessment using a swine animal model. Magn Reson Imaging 39:7-14

42. Sánchez-González J, Fernandez-Jiménez R, Nothnagel ND, López-Martín G, Fuster V, Ibañez B (2015) Optimization of dualsaturation single bolus acquisition for quantitative cardiac perfusion and myocardial blood flow maps. J Cardiovasc Magn Reson 17:21. https://doi.org/10.1186/s12968-015-0116-2

43. Fernández-Jiménez R, Sánchez-González J, Aguero J, del Trigo M, Galán-Arriola C, Fuster V, Ibáñez B (2015) Fast T2 gradientspin-echo (T2-GraSE) mapping for myocardial edema quantification: first in vivo validation in a porcine model of ischemia/reperfusion. J Cardiovasc Magn Reson 17:92. https://doi.org/10.1186/ s12968-015-0199-9

Publisher's Note Springer Nature remains neutral with regard to jurisdictional claims in published maps and institutional affiliations. 